\title{
Beneficial effects of the traditional medicine Igongsan and its constituent ergosterol on dextran sulfate sodium-induced colitis in mice
}

\author{
SU-JIN KIM ${ }^{1 *}$, HYUN-JI SHIN ${ }^{2 *}$, GEUN-HYUK LEE ${ }^{3 *}$, DAE-SEUNG KIM ${ }^{2}$, HYE-LIN KIM ${ }^{3}$, JINBONG PARK $^{3}$, \\ YUNU JUNG ${ }^{3}$, DONG-HYUN YOUN ${ }^{3}$, JONGWOOK KANG ${ }^{3}$, SEUNG-HEON HONG ${ }^{2}$ and JAE-YOUNG UM $^{3}$ \\ ${ }^{1}$ Department of Cosmeceutical Science, Daegu Hanny University, Yugok-dong, Kyungsan 712-715; \\ ${ }^{2}$ Department of Oriental Pharmacy, College of Pharmacy, Wonkwang Oriental Medicines Research Institute, \\ Iksan, Jeonbuk 570-749; ${ }^{3}$ Department of Pharmacology, College of Korean Medicine, Institute of Korean Medicine, \\ Kyung Hee University, Dongdaemun-Gu, Seoul 130-701, Republic of Korea
}

Received April 29, 2014; Accepted April 10, 2015

DOI: $10.3892 / \mathrm{mmr} .2015 .3824$

\begin{abstract}
Ulcerative colitis (UC) is a type of inflammatory bowel disease and is considered a chronic gastrointestinal disorder. Igongsan (IGS) is a Korean herbal medicine, which has been used to treat digestive disorders. However, the ameliorative effect and molecular mechanisms of IGS in intestinal inflammation have not yet been studied in detail. The present study aimed to investigate the protective effects of IGS and its constituent, ergosterol, in a mouse model of dextran sulfate sodium (DSS)-induced colitis. Colitis was induced in mice by supplementing their drinking water with $5 \%(\mathrm{w} / \mathrm{v})$ DSS for 7 days. The effects of IGS were then determined on DSS-induced clinical signs of colitis, including weight loss, colon shortening, diarrhea and obscure/gross bleeding. In addition, the effects of IGS were determined on the expression levels of inflammation-associated genes in the colon tissue of DSS-treated mice. The results of the present study demonstrated that mice treated with DSS exhibited marked clinical symptoms, including weight loss and reduced colon length. Treatment with IGS attenuated these symptoms and also suppressed the expression levels of tumor necrosis factor- $\alpha$ and interleukin-6, as well as the expression of cyclooxygenase- 2 in the colon tissue of DSS-treated mice. IGS also reduced the activation of the transcription factor nuclear factor- $\kappa \mathrm{B}$ p65 in the colon tissue of DSS-treated mice. In addition, ergosterol
\end{abstract}

Correspondence to: Professor Jae-Young Um, Department of Pharmacology, College of Korean Medicine, Institute of Korean Medicine, Kyung Hee University, 1 Hoegi-dong, Dongdaemun-gu, Seoul 130-701, Republic of Korea

E-mail: jyum@khu.ac.kr

${ }^{*}$ Contributed equally

Key words: ulcerative colitis, Igongsan, tumor necrosis factor- $\alpha$, interleukin- 6 , nuclear factor- $\kappa \mathrm{B}$ was shown to attenuate the DSS-induced clinical symptoms of colitis in mice. In conclusion, the present study provided experimental evidence that IGS may be a useful therapeutic drug for patients with UC.

\section{Introduction}

Ulcerative colitis (UC) is a common inflammatory intestinal disease, which belongs to a group of conditions known as inflammatory bowel diseases (IBD) (1). The pathogenesis of $\mathrm{UC}$ is believed to result from interactions between various genetic, immune and environmental factors (2). UC is associated with intestinal characteristics and symptoms, including weight loss, bloody diarrhea, fever and shortening of the colon (3). General features of tissue damage in UC include ulceration of the mucosa, blunting and loss of crypts, as well as infiltration of inflammatory cells (4). These features frequently result in epithelial dysplasia and DNA damage with microsatellite instability, which may result in cancer progression (5). Involvement of the entire colon for $>10$ years has been shown to predispose patients to colon cancer (5). In addition, it is well known that chronic inflammation of the colon contributes to colon carcinogenesis (6). To prevent the progression of cancer in patients with UC, an improved understanding of the pathogenesis of UC at the molecular and cellular level is required. UC is usually closely associated with intervals of acute exacerbation, and corticosteroid administration is effective for clinical remission (7). However, corticosteroids often have severe side effects, including hormonal disturbance, peptic ulcers, liver dysfunction and psychological issues (8). These adverse effects occasionally lead to discontinuation of corticosteroid treatment, which results in acute UC exacerbation (9). Therefore, alternative treatments for UC are required in order to help patients with UC avoid these clinical problems.

Recent studies have demonstrated that pro-inflammatory cytokines are associated with the initiation of colonic inflammation (10). Furthermore, it has been reported that mucosa from patients with UC exhibits increased expression levels of interleukin (IL)-6 (11,12). Mueller (13) previously detected 
high expression of tumor necrosis factor (TNF)- $\alpha$ in the blood, colonic tissue and stool samples of patients with UC (13). Therefore, in the field of UC treatment, there is a strong interest in developing agents that are able to block the generation or action of inflammatory cytokines.

Cyclooxygenase (COX) is a prostaglandin synthetase enzyme that is involved in the metabolism of arachidonic acid. There are two isoenzymes of COX: COX-1 and COX-2. COX-1 is expressed constitutively in the majority of tissues, particularly in the gastrointestinal tract, and is responsible for the production of prostaglandins that are associated with gastrointestinal integrity. Although COX-2 expression is low in the normal state, they are rapidly upregulated in response to various stimuli, including cytokines, growth factors, hormones and carcinogens. COX-2 is also responsible for producing prostaglandins associated with the mediation of inflammation (14). COX-2 expression has been shown to be increased in the mucosa of patients with UC $(15,16)$.

Nuclear factor (NF)- $\mathrm{\kappa B}$ is one of the most important transcription factors, which is responsible for the induction of genes that mediate innate and adaptive immunity. The role of NF- $\mathrm{KB}$ in UC has been investigated by numerous studies $(17,18)$. Ma et al (19) demonstrated that increases in intestinal epithelial tight-junction permeability require the activation of NF- $\mathrm{KB}$. In addition, increased DNA binding activity of NF- $\mathrm{KB}$, which is associated with high levels of IL-1 and IL-6, is observed in patients with UC (20). These studies suggested that the activation of NF- $\mathrm{KB}$ has a critical role in the initiation of intestinal inflammation in UC. Therefore, inhibiting the activation of NF- $\mathrm{kB}$ has been suggested as an effective anti-inflammatory strategy for the treatment of UC (21).

Traditional herbal medicine has been the subject of increased interest for its potential in the treatment of inflammation. Igongsan (IGS) is a Korean herbal medicine that contains Ginseng Radix, Atractylodis Rhizoma Alba, Poria Sclerotium, Glycyrrhizae Radix et Rhizoma and Citri Unshius Pericarpium. IGS is widely used to treat digestive disorders, including abdominal pain, dyspepsia and diarrhea. The use of IGS is based on knowledge from Traditional Korean Medicine (22), with few scientific studies available regarding the effects of IGS on intestinal inflammation supporting its clinical applications.

In order to support the traditional use of IGS by providing experimental evidence, the present study examined the effects of IGS and its constituent, ergosterol, on dextran sulfate sodium (DSS)-induced colitis. The aims of the present study were to assay the effects of IGS and ergosterol on the clinical signs of colitis, including weight loss, colon shortening, diarrhea and obscure/gross bleeding, and to investigate the effects of IGS and ergosterol on inflammatory-associated gene expression in DSS-treated colon tissue.

\section{Materials and methods}

Reagents. DSS (mol wt, 36,000-50,000) was purchased from MP Biomedicals North America (Solon, OH, USA). Mouse TNF- $\alpha$ affinity purified polyclonal, goat IgG (cat no. AF-410-NA), mouse TNF- $\alpha$ biotinylated affinity purified polyclonal, goat $\mathrm{IgG}$ (cat no. BF410) and recombinant mouse TNF- $\alpha$ (cat no. 410-MT) were purchased from R\&D Systems, Inc. (Minneapolis, MN,
Table I. Components of Igongsan.

Constituent

Amount (g)

Ginseng Radix

Atractylodis Rhizoma Alba

20

Poria Sclerotium

20

Glycyrrhizae Radix et Rhizoma

20

Citri Unshius Pericarpium

USA). Purified rat anti-mouse IL-6 (cat no. 55440), biotin rat anti-mouse IL-6 (cat no. 554402) and recombinant mouse IL-6 (cat no. 554582) were purchased from BD Biosciences (San Diego, CA, USA). Avidin peroxidase (cat no. A3151), ergosterol (cat no. E6510), sulfasalazine (cat no. S0883) and 2,2'-azinobis [3-ethylbenzothiazoline-6-sulfonic acid]-diammonium salt (ABTS; cat no. A9941) were purchased from Sigma-Aldrich (St. Louis, MO, USA). Hydrogen peroxide (cat no. 23150-0350) was purchased from Junsei Chemical Co., Ltd (Tokyo, Japan). COX-2 (cat no. sc-1745), NF- $\mathrm{B} / \mathrm{p} 65$ (cat no. sc-7151) and histone H1 (cat no. sc-10806) were purchased from from Santa Cruz Biotechnology, Inc. (Dallas, TX, USA). Peroxidase AffiniPure rabbit anti-goat IgG (H+L) (cat no. 305-035-003), peroxidase-conjugated AffiniPure goat anti-rabbit IgG (catalog no. 111-035-003) and peroxidase-conjugated AffiniPure goat anti-mouse IgG (catalog no. 115-035-062) antibodies were purchased from Jackson Immuno Research Laboratories, Inc. (West Grove, PA, USA).

Animals. Female BALB/c mice (four weeks old; body weight, 16-18 g) were purchased from the Dae-Han Experimental Animal Center (Eumsung, Korea). The mice $(n=48)$ were maintained in the College of Pharmacy, Wonkwang University (Iksan, Korea), and 5-10 mice were housed per cage in a laminar air-flow room. The atmosphere was maintained at a temperature of $22 \pm 1^{\circ} \mathrm{C}$ and relative humidity of $55 \pm 10 \%$ with a $12 \mathrm{~h}$ light/dark cycle throughout the study. Animal experimental procedures were approved by the Animal Ethics Committee of Wonkwang University (approval number WKU14-05).

Preparation of IGS. IGS is a herbal medicine composed of five herbs: Ginseng Radix (root of Panax ginseng, Araliaceae), Atractylodis Rhizoma Alba (rhizome of Atractylodes macrocephala, Compositae), Poria Sclerotium (sclerotium of Poria cocos, Polyporaceae), Glycyrrhizae Radix et Rhizoma (root and rhizome of Glycyrrhiza uralensis, Leguminosae) and Citri Unshius Pericarpium (Peel of Citrus unshiu, Rutaceae). The composition of IGS is shown in Table I. The components of IGS used in the present study were purchased from Daehak Oriental Drugstore (Iksan, Korea), and their identity was confirmed by Professor Seung-Heon Hong (Department of Oriental Pharmacy, Wonkwang University, Jeonbuk, Korea). The extract was prepared by decocting the dried prescription with boiling distilled water $(100 \mathrm{~g} / \mathrm{l})$. The extraction was decocted for $3 \mathrm{~h}$, and was then filtered and lyophilized prior to being maintained at $4^{\circ} \mathrm{C}$ (yield, $7.26 \%$ ). The samples were dissolved in distilled water and then filtered through a $0.22-\mu \mathrm{m}$ syringe filter (Merck Millipore, Carrigtwohill, Ireland). 
A

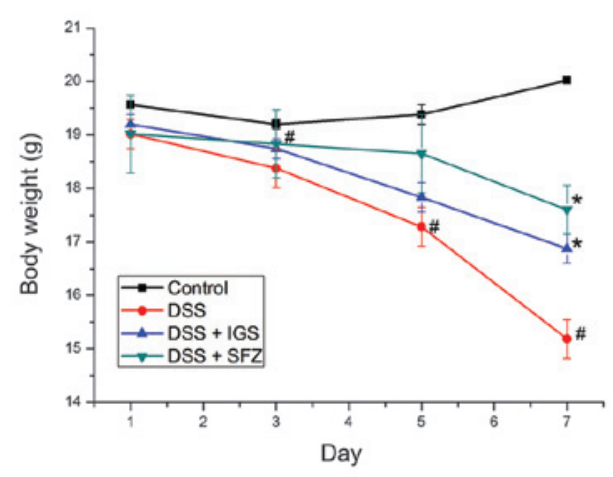

C

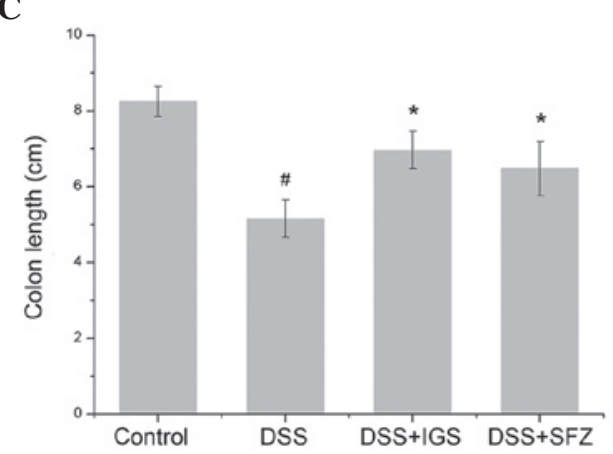

B

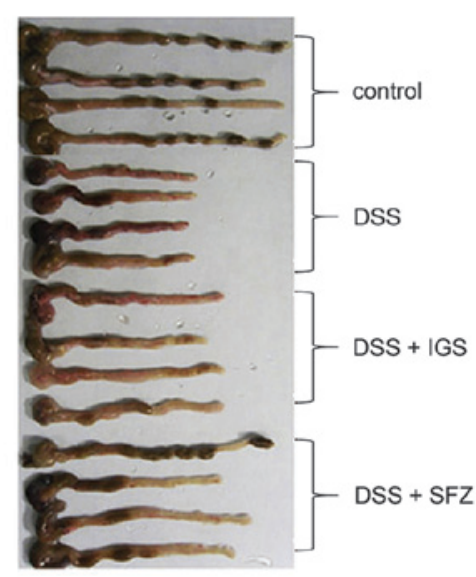

D

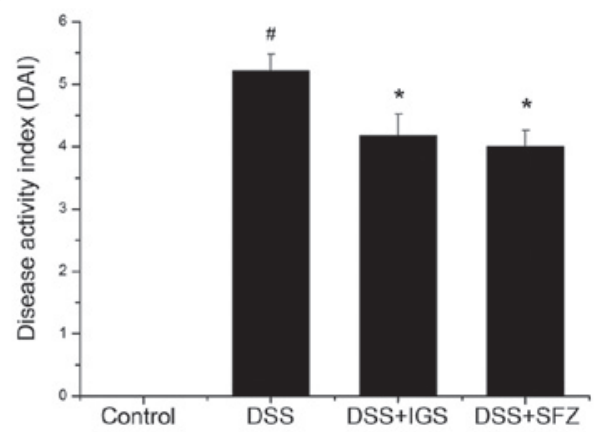

Figure 1. Effects of IGS on the clinical signs of DSS-induced colitis. Experimental colitis was induced in mice by supplementing their drinking water with 5\% (w/v) DSS for 7 days. IGS (100 mg/kg/day) was administered once a day for 7 days prior to intake of $5 \%$ DSS. (A) The body weight of the mice was measured. (B) Colons were harvested from the mice at day 7 after DSS treatment, and their length was measured. (C) Relative colon lengths are represented. (D) The DAI was calculated. SFZ $(150 \mathrm{mg} / \mathrm{kg}$ ) was used as a positive control. Values are presented as the mean \pm standard error of the mean (n=6) of duplicate determinations from three separate experiments. ${ }^{~} \mathrm{P}<0.05$ vs. control; " $\mathrm{P}<0.05$ vs. DSS alone. IGS, Igongsan; DSS, dextran sodium sulfate; DAI, disease activity index; SFZ, sulfasalazine.

Induction of colitis by DSS. Colitis was induced in mice as described in a previous study by our group (23). The mice were orally administered DSS dissolved in drinking water, which is a widely used experimental model of colitis. Briefly, acute colitis was induced in the mice by supplementing their drinking water with $5 \%(w / v)$ DSS for 7 days. The mice were checked daily for weight loss, stool consistency and the presence of gross bleeding. The mice were randomized into three groups $(n=6)$ : Mice receiving oral administration of IGS (100 mg/kg/day) or Ergosterol (20 mg/kg/day), mice treated with sulfasalazine (SFZ; $150 \mathrm{mg} / \mathrm{kg} /$ day) as a positive control, or mice treated with water as a negative control. IGS and SFZ were orally administered once a day for 7 days prior to DSS treatment. The mice were sacrificed by cervical dislocation and assessed after 7 days of treatment with DSS.

Disease activity index (DAI). The activity of intestinal disease was assessed through the following manifestations: Weight loss, diarrhea accompanied with blood and mucus, and shortening of the colon (3). In the present study, the DAI was obtained from the score of three major clinical symptoms: Weight loss, diarrhea and rectal bleeding, as described in a previous study by our group (23), based on a study by Murthy et al (24). Loss of body weight was calculated as the difference between initial and final weight. Diarrhea was defined by the absence of fecal pellet formation in the colon, and the presence of continuous fluid fecal material in the colon. The appearance of rectal bleeding was defined as diarrhea containing visible blood and gross rectal bleeding, and was scored as described for diarrhea. The DAI was calculated using the following formula: DAI $=($ weight loss score $)+($ diarrhea score $)+($ rectal bleeding score). The clinical parameters used here are comprehensive functional measures that are analogous to the subjective clinical symptoms observed in human UC (25). This method of scoring has been validated by repeated studies $(24,26)$.

Cytokine assay. A cytokine assay was performed using a modified ELISA as described previously (27). Tissue segments excised from distal colon were prepared by homogenization and extraction by PRO-PREP Protein Extraction solution (Intron Biotechnology, Inc., Seongnam, Korea). Following protein quantification (DC ${ }^{\mathrm{TM}}$ Protein Assay kit; Bio-Rad Laboratories, Inc., Hercules, CA, USA), equal protein concentrations of the tissue samples underwent ELISA. The ELISA was performed by coating 96 -well plates with mouse monoclonal antibodies targeting TNF- $\alpha$ and IL- 6 overnight at $4^{\circ} \mathrm{C}$. Assay plates were sequentially exposed to biotinylated mouse TNF- $\alpha$ and IL-6 for $1 \mathrm{~h}$, avidin peroxidase for $40 \mathrm{~min}$ and 2,2'-azinobis ABTS substrate solution containing $30 \% \mathrm{H}_{2} \mathrm{O}_{2}$. The plates were read at a wavelength of $405 \mathrm{~nm}$ with the VersaMax ${ }^{\mathrm{TM}}$ ELISA 
Microplate Reader (Molecular Devices, LLC, Sunnyvale, CA, USA).

Prostaglandin $E_{2}\left(P_{G} E_{2}\right)$ assay. The concentration of $\mathrm{PGE}_{2}$ in the colon tissue was measured by ELISA using a $\mathrm{PGE}_{2}$ assay kit (Stressgen Biotechnologies Corporation, San Diego, CA USA), according to the manufacturer's instructions. Duplicate aliquots of supernatant were measured for each sample.

Western blot analysis. The western blot analyses were performed as described previously (23). Briefly, the proteins were extracted from the distal colon tissue samples. The proteins were then separated using the Gradi-Gel 2 Gradient PAGE analysis kit (Elpis Biotech, Inc., DaeJeon, Korea) 7.5\% gel for SDS-PAGE prior to being transferred onto nitrocellulose membranes (GE Healthcare Bio-Sciences, Piscataway, NJ, USA). The membranes were then incubated with primary and secondary antibodies sequentially, and the protein bands were visualized using an Enhanced Chemiluminesence Detection system (GE Healthcare Bio-Sciences), according to the manufacturer's instructions. Relative protein levels were evaluated using GAPDH or histone as the reference protein in each condition. The band intensities were measured using ImageJ software, version 1.48 (National Institutes of Health, Bethesda, MD, USA).

Statistical analysis. Values are expressed as the mean \pm standard error of the mean of at least three experiments. The data were examined by one-way analysis of variance with Tukey's post hoc test. PASW Statistics software, version 18.0.0 (SPSS, Inc., Chicago, IL, USA) was used to conduct the statistical analysis. $\mathrm{P}<0.05$ was considered to indicate a statistically significant difference.

\section{Results}

IGS alleviates clinical symptoms of DSS-induced colitis. It has previously been reported that DSS-induced colitis in mice has a similar phenotype to human acute and chronic UC (28). In the present study, the inhibitory effects of IGS on the intestines of mice with DSS-induced experimental colitis were evaluated (Fig. 1). The physiological symptoms of colitis (weight loss, colon shortening, diarrhea and obscure/gross bleeding) were observed following 7 days of 5\% DSS treatment, and the DAIs of the mice were calculated. Mice treated with DSS exhibited significant weight loss $(15.19 \pm 0.37 \mathrm{~g}$; Fig. 1A) and colon shortening $(5.20 \pm 0.45 \mathrm{~cm})$ (Fig. 1B and C), as compared with the control group $(20.05 \pm 0.14 \mathrm{~g}$ and $8.23 \pm 0.34 \mathrm{~cm}$, respectively; $\mathrm{P}<0.05)$. The percentage of body weight loss and colon shortening was $\sim 26.01$ and $42 \%$, respectively. Mice treated with IGS had a significant attenuation of body weight loss $(16.88 \pm 0.28 \mathrm{~g})$ and colon shortening $(6.23 \pm 0.50 \mathrm{~cm})$ as compared with that in the mice with DSS-induced colitis $(\mathrm{P}<0.05)$ (Fig. 1A-C). In addition, the DAI was markedly decreased in the mice treated with IGS $(4.25 \pm 0.33)$ as compared with that in the mice treated with DSS alone $(5.22 \pm 0.25 ; \mathrm{P}<0.05)$ (Fig. 1D). SFZ is a drug widely used to treat colitis, which was used as the positive control in the present study; results for the SFZ group were similar to those of the IGS group within the error range.
A

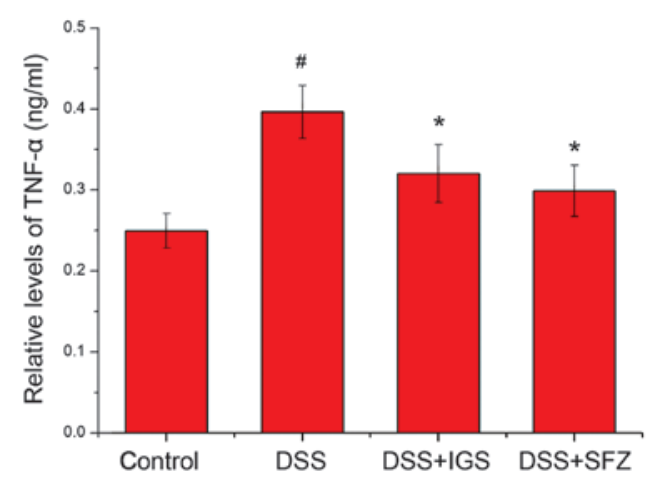

B

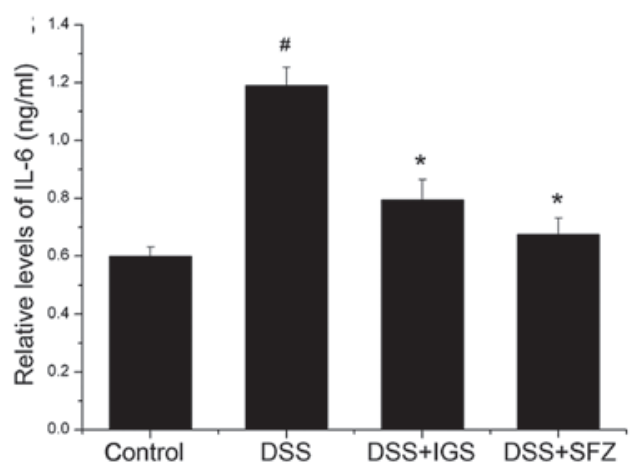

Figure 2. Effects of IGS on the levels of TNF- $\alpha$ and IL-6 in colon tissue from DSS-treated mice. Experimental colitis was induced in the mice by supplementing their drinking water with $5 \%(w / v)$ DSS for 7 days. IGS (100 mg/ $\mathrm{kg} /$ day) was administered once a day for 7 days prior to intake of $5 \%$ DSS. At the end of the experiment, the colon tissue was harvested and homogenized. (A and B) Levels of TNF- $\alpha$ and IL-6 in the indicated groups were measured by ELISA. Values are expressed as the mean \pm standard error of the mean $(n=6)$ of duplicate determinations from three separate experiments ${ }^{\#} \mathrm{P}<0.05$ vs. control; $\mathrm{P}<0.05$ vs. DSS alone. IGS, Igongsan; DSS, dextran sodium sulfate; SFZ, sulfasalazine; TNF, tumor necrosis factor; IL-6, interleukin-6.

IGS inhibits increases in TNF- $\alpha$ and IL-6 levels in DSS-induced colitis. The effects of IGS on TNF- $\alpha$ and IL-6 levels in colon tissue from mice with experimental colitis were determined. At the end of the experiment, the mouse colons were homogenized and examined using ELISA. As shown in Fig. 2A and B, the levels of TNF- $\alpha$ and IL-6 were significantly increased in the colon tissue of DSS-treated mice $(0.4 \pm 0.03$ and $1.2 \pm 0.06 \mathrm{ng} / \mathrm{ml})$ as compared with those in the control mice $(0.25 \pm 0.02$ and $0.6 \pm 0.04 \mathrm{ng} / \mathrm{ml} ; \mathrm{P}<0.05)$. However, administration of IGS reduced the DSS-induced increase in TNF- $\alpha$ and IL- 6 levels $(0.32 \pm 0.04$ and $0.8 \pm 0.07 \mathrm{ng} / \mathrm{ml}$, respectively; $\mathrm{P}<0.05)$. The rate of inhibition of TNF- $\alpha$ and IL- 6 by IGS was 21.71 and $33.34 \%$, respectively. In comparison, the rate of inhibition of TNF- $\alpha$ and IL-6 by SFZ was 25.01 and $36.11 \%$, respectively.

IGS inhibits increases in COX-2 expression and $P G E_{2}$ levels in DSS-induced colitis. The effects of IGS on the protein expression levels of COX-2 were determined by western blot analysis. The expression levels of COX-2 were significantly increased in colon tissue of mice treated with DSS $(0.88 \pm 0.10)$ as compared with those in the control mice $(0.46 \pm 0.08 ; \mathrm{P}<0.05)$. However, treatment with IGS reduced the expression levels of COX-2 in the DSS-treated mice $(0.66 \pm 0.11 ; \mathrm{P}<0.05)$ (Fig. $3 \mathrm{~A}$ and $\mathrm{B})$. 
A

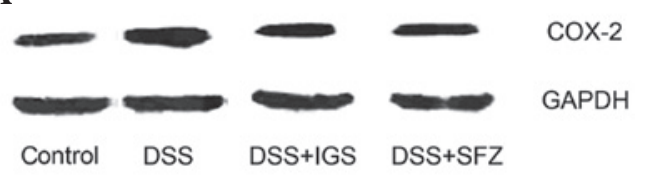

B
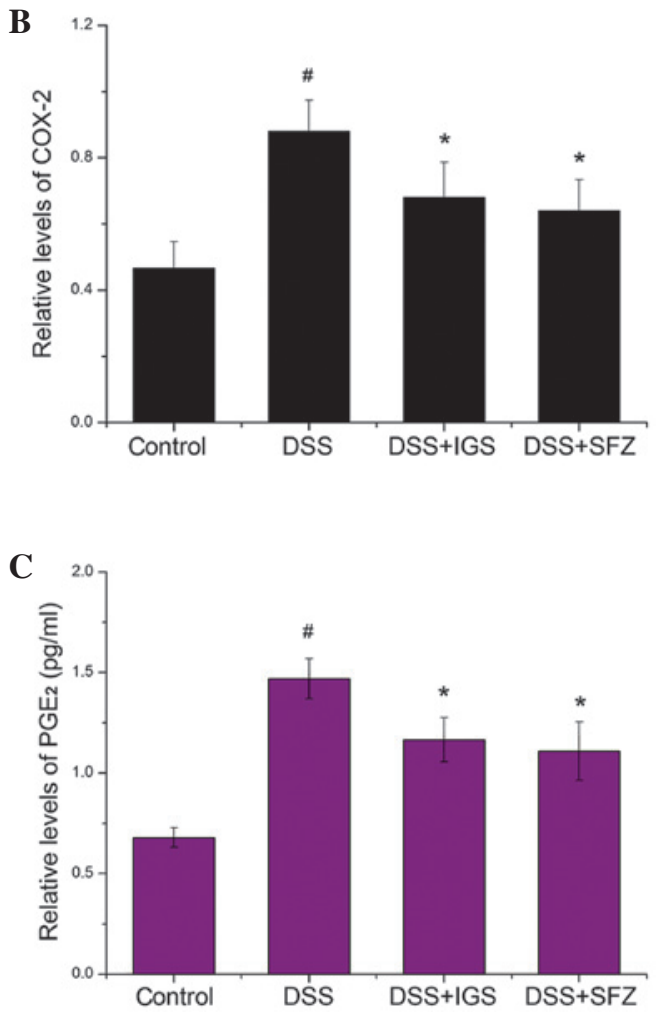

Figure 3. Effects of IGS on the expression of COX-2 and levels of $\mathrm{PGE}_{2}$ in colon tissue from DSS-treated mice. Experimental colitis was induced in mice by supplementing their drinking water with 5\% (w/v) DSS for 7 days. IGS $(100 \mathrm{mg} / \mathrm{kg} / \mathrm{day})$ was administered once a day for 7 days prior to intake of 5\% DSS. At the end of the experiment, colon tissue was harvested and homogenized. (A) Protein expression levels of COX-2 were determined by western blotting. (B) Relative expression levels of COX-2 from quantitative analysis of A. (C) Levels of $\mathrm{PGE}_{2}$ in the indicated groups were evaluated using an immunoassay kit. Values are expressed as the mean \pm standard error of the mean $(n=6)$ of duplicate determinations from three separate experiments. ${ }^{~} \mathrm{P}<0.05$ vs. control; ${ }^{*} \mathrm{P}<0.05$ vs. DSS alone. IGS, Igongsan; DSS, dextran sodium sulfate; SFZ, sulfasalazine; COX-2, cyclyooxygenase-2; $\mathrm{PGE}_{2}$, prostaglandin E2.

COX-2 catalyzes the biosynthesis of $\mathrm{PGE}_{2}$; therefore, the present study determined whether IGS exerted an effect on the levels of $\mathrm{PGE}_{2}$ in colon tissue. As shown in Fig. 3C, $\mathrm{PGE}_{2}$ levels were enhanced in response to DSS $(1.46 \pm 0.10 \mathrm{pg} / \mathrm{ml})$; however, this increase was significantly inhibited by IGS $(1.16 \pm 0.10 \mathrm{pg} / \mathrm{ml} ; \mathrm{P}<0.05)$. The rate of inhibition of $\mathrm{PGE}_{2}$ production by IGS $(100 \mathrm{mg} / \mathrm{kg} /$ day $)$ was $32.22 \%$.

IGS suppresses the activation of NF- $\kappa B$ p 65 in DSS-induced colitis. Activation of NF- $\mathrm{KB}$ p65 is involved in colitis (18); therefore, inhibition of NF- $\kappa \mathrm{B}$ activation has been suggested as an anti-inflammatory therapeutic strategy for colitis. The present study investigated whether IGS regulated the activa-

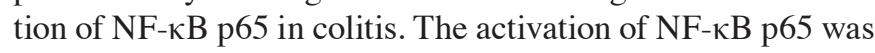

A
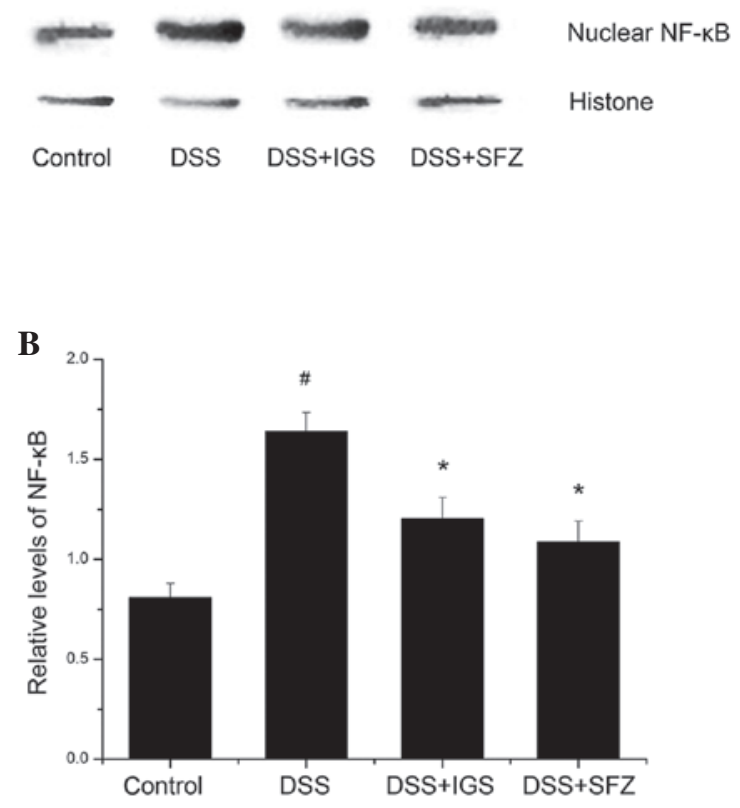

Figure 4. Effects of IGS on the activation of NF- $\mathrm{NB}$ p 65 in colon tissue from DSS-treated mice. Experimental colitis was induced in mice by supplementing their drinking water with $5 \%(\mathrm{w} / \mathrm{v})$ DSS for 7 days. IGS (100 mg/kg/day) was administered once a day for 7 days prior to $5 \%$ DSS supplement. (A) At the end of the experiment, colon tissue was harvested and homogenized. Nuclear extracts were isolated from the colon tissue and evaluated for NF- $\kappa \mathrm{B} / \mathrm{p} 65$ by western blot analysis. (B) Relative expression levels of $\mathrm{NF}-\kappa \mathrm{B}$ were measured using an image analyzer. Values are expressed as the mean \pm standard error of the mean $(n=6)$ of duplicate determinations from three separate experiments. ${ }^{\#} \mathrm{P}<0.05$ vs. control; ${ }^{*} \mathrm{P}<0.05$ vs. DSS alone. IGS, Igongsan; DSS, dextran sodium sulfate; SFZ, sulfasalazine; $\mathrm{NF}-\kappa \mathrm{B}$; nuclear factor- $\kappa \mathrm{B}$.

increased in the colon tissue of the DSS group $(1.70 \pm 0.09)$ as compared with that in the control group $(0.83 \pm 0.08 ; \mathrm{P}<0.05)$. However, oral IGS administration significantly suppressed the DSS-induced activation of NF- $\mathrm{KB}$ p65 in colon tissue $(1.23 \pm 0.10 ; \mathrm{P}<0.05)$ (Fig. 4).

Ergosterol alleviates clinical symptoms of DSS-induced colitis. Ergosterol is a component of IGS. The present study evaluated the inhibitory effects of ergosterol on DSS-induced colitis. As shown in Figs. 5A-D, ergosterol significantly inhibited DSS-induced weight loss, colon shortening and DAI in the mice (DSS vs. DSS + Ergo: $16.08 \pm 0.49 \mathrm{~g}$ vs. $17.45 \pm 0.58 \mathrm{~g}$; $5.40 \pm 0.23 \mathrm{~cm}$ vs. $6.32 \pm 0.16 \mathrm{~cm}$; and $2.25 \pm 0.57$ vs. $1.75 \pm 0.32$ ) $(\mathrm{P}<0.05)$. In addition, it was observed that ergosterol attenuated DSS-induced activation of NF- $\kappa \mathrm{B}$ p65 in colon tissue from mice with experimental colitis (DSS vs. DSS + Ergo, $1.32 \pm 0.10$ vs. $1.07 \pm 0.11 ; \mathrm{P}<0.05$ ) (Fig. $5 \mathrm{E}$ and $\mathrm{F}$ ).

\section{Discussion}

Traditional herbal medicines have been used effectively for centuries, based on traditional knowledge; however, the pharmacological mechanisms of action of these herbal prescriptions have yet to be fully elucidated. The present study aimed to provide experimental evidence regarding the use of IGS as a potential therapeutic drug for patients with UC. The present study demonstrated that IGS is able to inhibit inflammation 


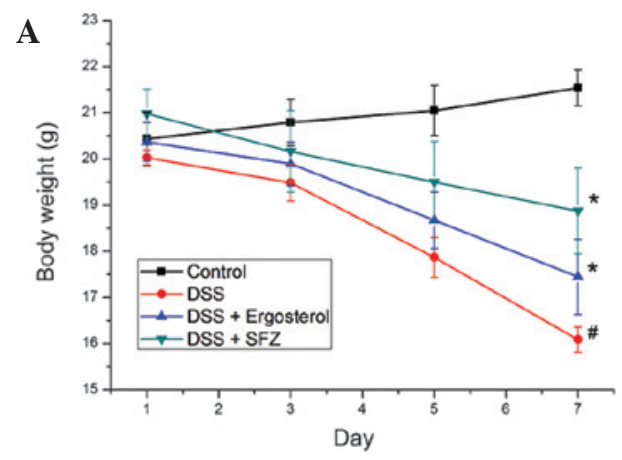

C

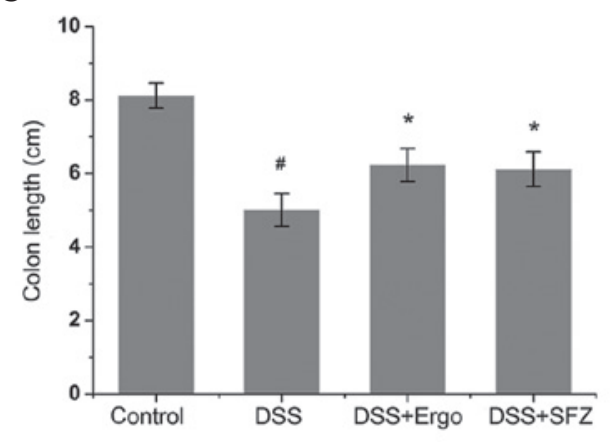

$\mathbf{E}$

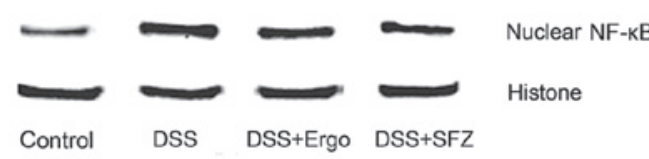

B

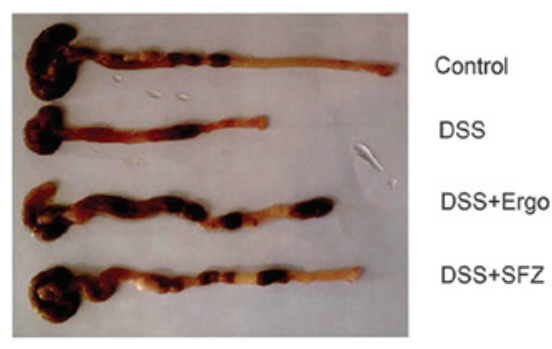

D

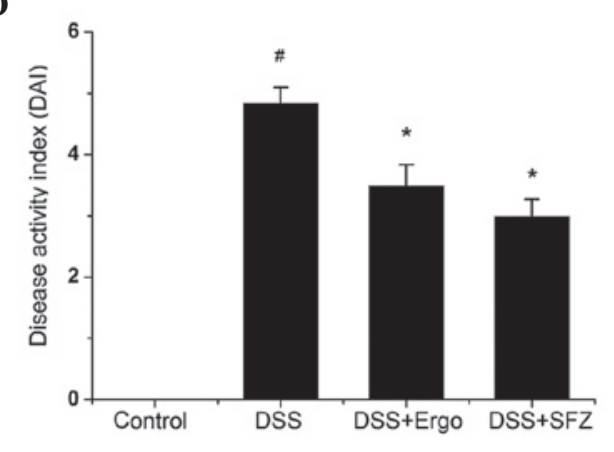

$\mathbf{F}$

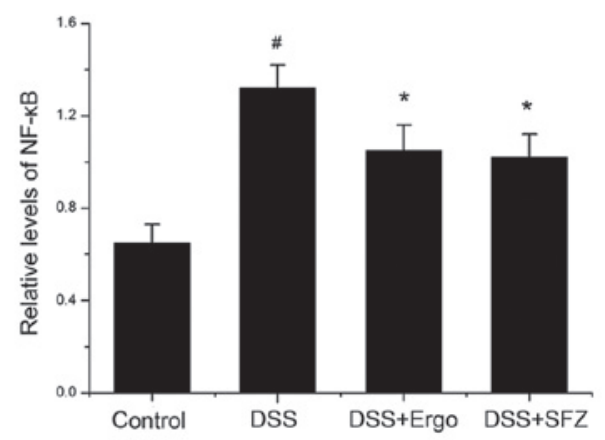

Figure 5. Effects of ergosterol on the clinical characteristics of DSS-induced colitis. Experimental colitis was induced in mice by supplementing their drinking water with $5 \%$ (w/v) DSS for 7 days. Ergosterol $(20 \mathrm{mg} / \mathrm{kg}$ ) was administered once a day for 7 days prior to intake of $5 \%$ DSS. (A) Body weight of the mice was measured. (B) Colons were harvested at day 7 after DSS treatment, and their length was measured. (C) Relative colon lengths are presented. (D) The DAI was calculated. (E) Nuclear extracts were isolated from the colon tissue and evaluated for NF- $\mathrm{\kappa B} / \mathrm{p} 65$ expression. (F) Relative expression levels of NF- $\mathrm{kB}$. SFZ $(150 \mathrm{mg} / \mathrm{kg})$ was used as a positive control. Values are expressed as the mean \pm standard error of the mean $(\mathrm{n}=6)$ of duplicate determinations from three separate experiments. ${ }^{~} \mathrm{P}<0.05$ vs. control; "P<0.05 vs. DSS alone. IGS, Igongsan; DSS, dextran sodium sulfate; SFZ, sulfasalazine; DAI, disease activity index; $\mathrm{NF}-\kappa \mathrm{B}$; nuclear factor- $\kappa \mathrm{B}$.

and colon injury provoked by DSS treatment. The results indicated that there is an important molecular mechanism by which IGS ameliorates intestinal inflammation.

$\mathrm{UC}$ is a typical inflammatory disease of the intestine, which belongs to a group of conditions known as IBD. However, despite numerous years of extensive research implicating immune dysfunction, genetic susceptibility and bacterial flora within the intestinal environment as possible factors associated with the development of the disease (29), its pathogenesis has remained to be elucidated. The signs and symptoms of UC include abdominal pain, weight loss and diarrhea accompanied with bleeding (30-32). Therapies for UC include glucocorticosteroids and SFZ $(33,34)$; however, these treatments often have severe adverse effects. Therefore, the requirement for anti-inflammatory agents with fewer side effects is growing. Recently, traditional herbal medicine has received increased interest regarding the treatment of such disorders. Previous studies by our group have demonstrated that various herbal medicines have anti-colitis effects in mice $(23,35)$. The present study investigated the effects of IGS on UC, using the DSS-induced mouse model of colitis. Treatment with IGS reduced DSS-induced weight loss and colon shortening. In addition, the DAI, which is a scale determined by measuring the levels of three major clinical symptoms (weight loss, diarrhea and rectal bleeding), was markedly inhibited in the group treated with IGS. The protective effect of IGS on colon shortening and DAI was similar to the protective effect of SFZ. These results therefore suggested that IGS may effectively inhibit the symptoms of DSS-induced colitis. 
Pro-inflammatory cytokines are closely associated with the initiation of the inflammatory response in colitis. It has previously been shown that TNF- $\alpha$ is strongly associated with the pathogenesis and progression of intestinal inflammatory disorders (36). It has also been reported that levels of IL-6 are markedly elevated in patients with UC, and that IL-6 has an integral role in its pathogenesis (37). Therefore, studies on novel biological therapies for UC have focused on blocking components of the inflammatory cascade, such as cytokines. COX-2 is also closely associated with numerous pathological processes, including UC. DSS elicits COX-2 expression to regulate the production of prostaglandins, which induce gastrointestinal fluid secretion in the inflammatory pathway (38). The present study demonstrated that the levels of TNF- $\alpha$, IL- 6 and COX-2 were increased in the colon tissue of DSS-treated mice as compared with those in the control mice, and treatment with IGS reduced these levels. In addition, the inhibitory effect of IGS on the increases in TNF- $\alpha$, IL-6 and COX-2 levels in DSS-treated colon tissue was similar to the effect of SFZ. These results indicated that the anti-inflammatory effects of IGS may be attributed to the regulation of inflammatory mediators in DSS-induced colitis.

$\mathrm{NF}-\kappa \mathrm{B}$ is an important transcription factor, particularly for the activation of numerous inflammatory mediators, cytokines including IL-1 $\beta$ and IL-6, and COX-2. NF- $\kappa$ B p65 has been reported to be a critically important factor in chronic inflammatory diseases (39). Therefore, $N F-\kappa B$ is considered an ideal target for a molecular therapy of UC, and extensive efforts have been made to develop novel treatments targeting $\mathrm{NF}-\kappa \mathrm{B}(17,40,41)$. In the present study, it was observed that the activation of $\mathrm{NF}-\kappa \mathrm{B}$ p 65 was significantly increased in the colon tissue of DSS-treated mice as compared with that in the control group, and this increase was attenuated by treatment with IGS. These results indicated that the mechanism of action of IGS is a novel mechanism involving the regulation of NF- $\kappa \mathrm{B}$ p65 activation in DSS-induced colitis. Therefore, it may be hypothesized that IGS exerts its anti-inflammatory effect in UC by regulating the activation of NF- $\kappa \mathrm{B}$.

IGS is composed of five herbs, each of which have been reported to have various effects; in particular, ginsenoside Rb1, Rd and Re of Ginseng Radix (42-44), hesperidin of Unshius Pericarpium (45), and glycyrrhizic acid of Glycyrrhizae Radix et Rhizoma (46) have been shown to exhibit anti-colitis effects. Ergosterol is a compound present in Poria Sclerotium. It has previously been reported that ergosterol may be beneficial in the treatment of the allergic response by decreasing immunoglobulin E in mucosal-type mast cells (47). However, there is currently no information available regarding the effects of ergosterol on colitis. Therefore, an aim of the present study was to evaluate the possible modulating effects of ergosterol on colitis. The results of the present study demonstrated that ergosterol was able to attenuate clinical symptoms of DSS-induced colitis in mice.

In conclusion, the present study demonstrated that treatment with IGS significantly reduced the clinical symptoms and the levels of inflammatory mediators in a DSS-induced murine model of colitis. These results suggested that IGS may be a useful therapeutic candidate for colitis. However, further studies are required to reveal the precise mechanisms underlying the effects of IGS in intestinal inflammatory disorders.

\section{Acknowledgements}

The present study was supported by the National Research Foundation of Korea grant funded by the Korean government (MSIP) (grant nos. 2011-0010435, 2013R1A2A2A03006068, 2012-0007669 and 2011-0030130).

\section{References}

1. Hyams JS: Inflammatory bowel disease. Pediatr Rev 21: 291-295, 2000.

2. Danese S, Sans M and Fiocchi C: Inflammatory bowel disease: The role of environmental factors. Autoimmun Rev 3: 394-400, 2004.

3. Hendrickson BA, Gokhale R and Cho JH: Clinical aspects and pathophysiology of inflammatory bowel disease. Clin Microbiol Rev 15: 79-94, 2002.

4. Lichtenstein GR and Rutgeerts P: Importance of mucosal healing in ulcerative colitis. Inflamm Bowel Dis 16: 338-346, 2010.

5. Ullman T, Croog V, Harpaz N, Sachar D and Itzkowitz S: Progression of flat low-grade dysplasia to advanced neoplasia in patients with ulcerative colitis. Gastroenterology 125: 1311-1319, 2003.

6. Eaden JA, Abrams KR and Mayberry JF: The risk of colorectal cancer in ulcerative colitis: A meta-analysis. Gut 48: 526-535, 2001.

7. Domènech E: Inflammatory bowel disease: Current therapeutic options. Digestion 73 Suppl 1: 67-76, 2006.

8. Buchman AL: Side effects of corticosteroid therapy. J Clin Gastroenterol 33: 289-294, 2001.

9. Farrell RJ and Kelleher D: Glucocorticoid resistance in inflammatory bowel disease. J Endocrinol 178: 339-346, 2003.

10. Li Y, de Haar C, Chen M, et al: Disease-related expression of the IL6/STAT3/SOCS3 signalling pathway in ulcerative colitis and ulcerative colitis-related carcinogenesis. Gut 59: 227-235, 2010.

11. Papadakis KA and Targan SR: Role of cytokines in the pathogenesis of inflammatory bowel disease. Annu Rev Med 51: 289-298, 2000.

12. Ogata $\mathrm{H}$ and Hibi $\mathrm{T}$ : Cytokine and anti-cytokine therapies for inflammatory bowel disease. Curr Pharm Des 9: 1107-1113, 2003.

13. Mueller C: Tumour necrosis factor in mouse models of chronic intestinal inflammation. Immunology 105: 1-8, 2002.

14. Vane JR, Bakhle YS and Botting RM: Cyclooxygenases 1 and 2. Annu Rev Pharmacol Toxicol 38: 97-120, 1998.

15. Roberts PJ, Morgan K, Miller R, Hunter JO and Middleton SJ: Neuronal COX-2 expression in human myenteric plexus in active inflammatory bowel disease. Gut 48: 468-472, 2001.

16. Agoff SN, Brentnall TA, Crispin DA, et al: The role of cyclooxygenase 2 in ulcerative colitis-associated neoplasia. Am J Pathol 157: 737-745, 2000.

17. Bantel H, Berg C, Vieth M, Stolte M, Kruis W and Schulze-Osthoff K: Mesalazine inhibits activation of transcription factor NF-kappaB in inflamed mucosa of patients with ulcerative colitis. Am J Gastroenterol 95: 3452-3457, 2000.

18. Andresen L, Jørgensen VL, Perner A, Hansen A, Eugen-Olsen J and Rask-Madsen J: Activation of nuclear factor kappaB in colonic mucosa from patients with collagenous and ulcerative colitis. Gut 54: 503-509, 2005.

19. Ma TY, Iwamoto GK, Hoa NT, et al: TNF-alpha-induced increase in intestinal epithelial tight junction permeability requires NF-kappa B activation. Am J Physiol Gastrointest Liver Physiol 286: G367-G376, 2004.

20. Atreya I, Atreya R and Neurath MF: NF-kappaB in inflammatory bowel disease. J Intern Med 263: 591-596, 2008.

21. Jobin C and Sartor RB: The I kappa B/NF-kappa B system: A key determinant of mucosalinflammation and protection. Am J Physiol Cell Physiol 278: C451-462, 2000.

22. Kim SJ, Shin HJ, Lee BJ, et al: The antiinflammatory mechanism of Igongsan in mouse peritoneal macrophages via suppression of NF- $\mathrm{B}$ /Caspase-1 activation. Phytother Res 28: 736-744, 2014.

23. Kim SJ, Kim YG, Kim DS, et al: Oldenlandia diffusa ameliorates dextran sulphate sodium-induced colitis through inhibition of NF- $\kappa$ B activation. Am J Chin Med 39: 957-969, 2011.

24. Murthy SN, Cooper HS, Shim H, Shah RS, Ibrahim SA and Sedergran DJ: Treatment of dextran sulfate sodium-induced murine colitis by intracolonic cyclosporin. Dig Dis Sci 38: 1722-1734, 1993 
25. Cooper HS, Murthy SN, Shah RS and Sedergran DJ: Clinicopathologic study of dextran sulfate sodium experimental murine colitis. Lab Invest 69: 238-249, 1993.

26. Porter SN, Howarth GS and Butler RN: An orally administered growth factor extract derived from bovine whey suppresses breath ethane in colitic rats. Scand J Gastroenterol 33: 967-974, 1998.

27. Kim MS, Lim WK, Cha JG, et al: The activation of PI 3-K and PKC zeta in PMA-induced differentiation of HL-60 cells. Cancer Lett 171: 79-85, 2001.

28. Okayasu I, Hatakeyama S, Yamada M, Ohkusa T, Inagaki Y and Nakaya R: A novel method in the induction of reliable experimental acute and chronic ulcerative colitis in mice. Gastroenterology 98: 694-702, 1990.

29. Fiocchi C: Inflammatory bowel disease: Evolutionary concepts in biology, epidemiology, mechanisms and therapy. Curr Opin Gastroenterol 29: 347-349, 2013.

30. Ardizzone S and Bianchi Porro G: Biologic therapy for inflammatory bowel disease. Drugs 65: 2253-2286, 2005.

31. Rufo PA and Bousvaros A: Current therapy of inflammatory bowel disease in children. Paediatr Drugs 8: 279-302, 2006

32. Sato K, Ohkura S, Kitahara Y, et al: Involvement of CPI-17 downregulation in the dysmotility of the colon from dextran sodium sulphate-induced experimental colitis in a mouse model. Neurogastroenterol Motil 19: 504-514, 2007.

33. Sandborn WJ and Targan SR: Biologic therapy of inflammatory bowel disease. Gastroenterology 122: 1592-1608, 2002.

34. Ishiguro $\mathrm{K}$, Ando $\mathrm{T}$, Maeda $\mathrm{O}$, et al: Paeonol attenuates TNBS-induced colitis by inhibiting NF-kappaB and STAT1 transactivation. Toxicol Appl Pharmacol 217: 35-42, 2006.

35. Kim SJ, Kim KW, Kim DS, et al: The protective effect of Cassia obtusifolia on DSS-induced colitis. Am J Chin Med 39: 565-577, 2011

36. Raddatz D, Bockemühl $\mathrm{M}$ and Ramadori G: Quantitative measurement of cytokine mRNA in inflammatory bowel disease: Relation to clinical and endoscopic activity and outcome. Eur J Gastroenterol Hepatol 17: 547-557, 2005.
37. Zheng P, Niu FL, Liu WZ, Shi Y and Lu LG: Anti-inflammatory mechanism of oxymatrine in dextran sulfate sodium-induced colitis of rats. World J Gastroenterol 11: 4912-4915, 2005.

38. Beubler E, Schuligoi R, Chopra AK, Ribardo DA and Peskar BA: Cholera toxin induces prostaglandin synthesis via post-transcriptional activation of cyclooxygenase-2 in the rat jejunum. J Pharmacol Exp Ther 297: 940-945, 2001.

39. Tak PP and Firestein GS: NF-kappaB: A key role in inflammatory diseases. J Clin Invest 107: 7-11, 2001.

40. Bak YK, Lampe JW and Sung MK: Effects of dietary supplementation of glucosamine sulfate on intestinal inflammation in a mouse model of experimental colitis. J Gastroenterol Hepatol 29: 957-963, 2014.

41. Song YA, Park YL, Kim KY, et al: Black tea extract prevents lipopolysaccharide-induced NF- $\kappa \mathrm{B}$ signaling and attenuates dextran sulfate sodium-induced experimental colitis. BMC Complement Altern Med 11: 91, 2011.

42. Joh EH, Lee IA, Jung IH and Kim DH: Ginsenoside Rb1 and its metabolite compound K inhibit IRAK-1 activation - the key step of inflammation. Biochem Pharmacol 82: 278-286, 2011.

43. Yang XL, Guo TK, Wang YH, et al: Ginsenoside Rd attenuates the inflammatory response via modulating p38 and JNK signaling pathways in rats with TNBS-induced relapsing colitis. Int Immunopharmacol 12: 408-414, 2012.

44. Lee IA, Hyam SR, Jang SE, Han MJ and Kim DH: Ginsenoside Re ameliorates inflammation by inhibiting the binding of lipopolysaccharide to TLR4 on macrophages. J Agric Food Chem 60: 9595-9602, 2012

45. Xu L, Yang ZL, Li P and Zhou YQ: Modulating effect of Hesperidin on experimental murine colitis induced by dextran sulfate sodium. Phytomedicine 16: 989-995, 2009.

46. Liu Y, Xiang J, Liu M, Wang S, Lee RJ and Ding H: Protective effects of glycyrrhizic acid by rectal treatment on a TNBS-induced rat colitis model. J Pharm Pharmacol 63: 439-446, 2011.

47. Kageyama-Yahara N, Wang P, Wang X, et al: The inhibitory effect of ergosterol, a bioactive constituent of a traditional Japanese herbal medicine saireito on the activity of mucosal-type mast cells. Biol Pharm Bull 33: 142-145, 2010. 